\title{
Double-door Laminoplasty by Splitting Spinous Processes
}

\author{
Atsushi Seichi, Katsushi Takeshita, Hiroshi Kawaguchi, and Kozo Nakamura
}

\section{Concept of Double-door Laminoplasty}

Until the 1970s, laminectomy had been the sole therapeutic option for posterior decompression of the spinal cord. However, wide laminectomy of the cervical spine sometimes caused early or late neurological deterioration (or both). The possible causes of such deterioration were the progression of malalignment such as kyphosis or listhesis, postoperative progression of ossification of the posterior longitudinal ligament (OPLL) associated with malalignment and instability of the cervical spine in patients with OPLL, and massive scar formation in the epidural space, known as "postlaminectomy membrane" [1-4]. To resolve the problems associated with laminectomy, several variations of laminoplasty have been developed in Japan and used instead of laminectomy [5-7].

One of the most popular laminoplasties of the bilateral hinge type is double-door laminoplasty via the mid-sagittal splitting method, which was devised in 1980 by Takahide Kurokawa (Professor Emeritus of Tokyo University). A preliminary report on doubledoor laminoplasty was published in 1982 [8]. The main aim of this laminoplasty was to expand the spinal canal symmetrically while preserving the mobility of the cervical spine. In common with other kinds of laminoplasty, the aims of this procedure to preserve the posterior structure in the midline of the cervical spine were to prevent postoperative progression of malalignment and instability of the cervical spine and to protect the spinal cord from postlaminectomy membrane. Compared with Z-laminoplasty, double-door laminoplasty is technically straightforward; and compared with unilateral hinge-type laminoplasty, such as Hirabayashi's method, double-door laminoplasty has some theoretical and practical advantages: symmetrical expansion of the spinal canal and avoidance of hemor-

Department of Orthopaedic Surgery, The University of Tokyo, 7-3-1 Hongo, Bunkyo-ku, Tokyo 113-8655, Japan rhage from the epidural veins because of the limited number of these veins in the midline.

Because the concept of laminoplasty has been widely accepted in Japan and recently in the United States and Europe, laminectomy without fusion is now rarely performed. However, there have been only a limited number of comparative studies on laminectomy versus laminoplasty $[9,10]$. We should be aware that there is criticism of the superiority of laminoplasty over laminectomy [11] because of a lack of randomized control trials.

In this chapter we describe the technical points of double-door laminoplasty and offer retrospective reviews of the surgical results.

\section{Indications}

Cervical myelopathy caused by multisegmental stenotic conditions due to OPLL of a continuous or mixed type is an indication for double-door laminoplasty. This method is also indicated for patients with localized OPLL combined with developmental spinal canal stenosis. Because the mechanism is posterior decompression of the spinal cord, the kyphotic cervical spine is theoretically not a good indication [12]. However, when the kyphosis is not severe, positive surgical effects can be expected [13]. The surgical effects for patients with a severely kyphotic cervical spine remain unknown. In our recent study using thin-slice computed tomography (CT) scanning and intraoperative ultrasonography, OPLL thickness of more than $7 \mathrm{~mm}$ was the morphological limitation of posterior decompression, but it has not been proved to cause a poor result [14].

The Japanese Orthopaedic Association (JOA) score has been employed to determine the indications for this surgery. Many surgeons used a JOA score below 13/17 as an indication. However, the ratings of this system are not linear, and it is not a good idea to make a decision based on a total score. A motor score for either or both upper and lower extremities of 2 or less is an indication for surgery. However, in relatively young patients with long tract signs and a T2-weighted magnetic resonance 
imaging (MRI) high-intensity area in the spinal cord due to severe cord compression, mild myelopathy is also an indication, especially to prevent acute spinal cord injury after a fall.

\section{Procedure}

The extent of the laminoplasty is usually from C3 to $\mathrm{C} 7$, although if there is OPLL up to $\mathrm{C} 2$ or $\mathrm{C} 1$ these levels are included as well. Preoperative MRI is helpful for determining the laminoplasty level. The patient is placed on a modified Hall frame in a prone position. The neck is in a neutral position or slightly flexed. A midline skin incision is employed after administration of epinephrine. A posterior approach is made along the edge of the nuchal ligament to the line of the spinous processes (Fig. 1). By using this approach we are trying to maintain the continuity of the nuchal ligament because a biomechanical study using a cadaver model suggested that injury to this ligament may increase the risk of cervical spine instability and malalignment [15]. The semispinalis cervicis is not necessarily detached from the C2 spinous process when the area of laminoplasty is below C3. However, for a laminoplasty that includes $\mathrm{C} 2$, the semispinalis cervicis is temporarily detached from the $\mathrm{C} 2$ spinous process. In a case where the surgical field extends to $\mathrm{C} 1$ and $\mathrm{C} 2$, the rectus capitis posterior major and

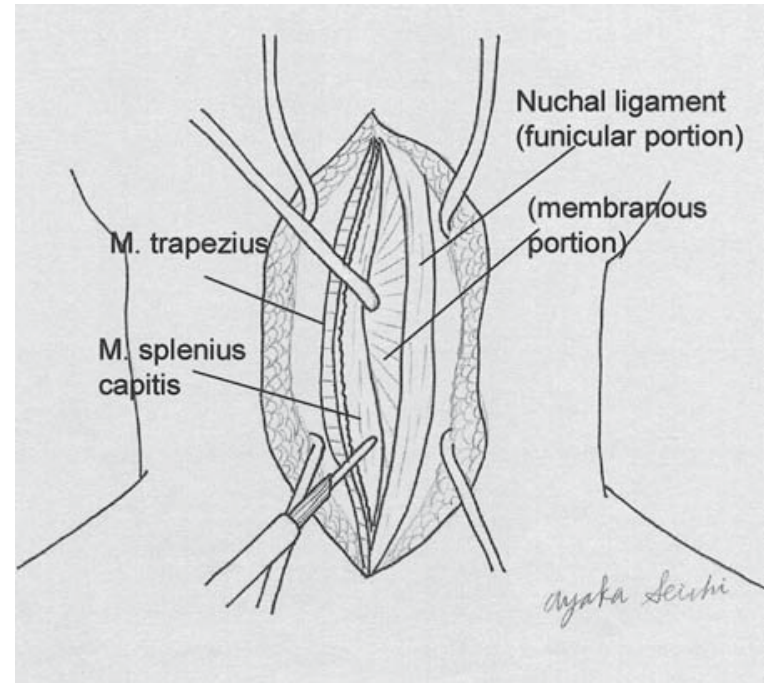

Fig. 1. Posterior approach to the cervical laminae. The approach is made along the edge of the nuchal ligament. The most superficial muscle, the trapezius, is separated from the superior nuchal ligament. The splenius capitis muscles are also detached from the nuchal ligament. This approach reserves the continuity of the nuchal ligament obliqus capitis inferior muscles have been detached, but recently we have performed $\mathrm{C} 1$ and C2 laminoplasties without detaching these two muscles (Fig. 2). The cervical laminae are exposed laterally to the medial border of the facet joint, and interspinous ligaments and muscles are removed using a rongeur. The spinous processes are sagittally split to the base with a $2 \mathrm{~mm}$ diameter steel burr, and the separation is completed with a 2 or $3 \mathrm{~mm}$ diameter diamond burr. After bilateral troughs (grooves) for the hinges are carefully made with a $3-4 \mathrm{~mm}$ diameter diamond burr at the transitional area between the facet joint and laminae, spinal canal enlargement is achieved by opening the split laminae bilaterally with a specially designed laminae spreader (TACT Medical, Tokyo, Japan) (Fig. 2). Preoperative $C T$ is helpful for determining the exact position of the lateral troughs. These troughs should not be made too medial or too lateral. Troughs that are too medial allow insufficient enlargement of the spinal canal, which causes constriction of the dural tube between the split spinous processes; and troughs that are too lateral make opening the split laminae difficult. The surgeon should confirm that the hinges preserve their elasticity and that the opening of the split laminae is symmetrical. Care should be taken not to make the troughs too deep, which makes the laminae unstable.

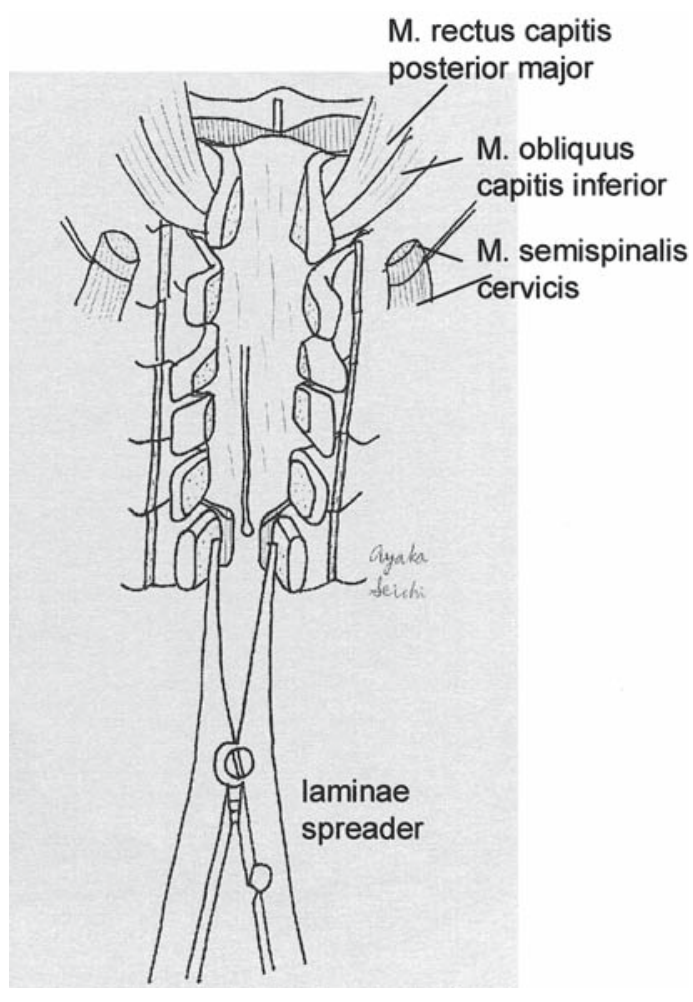

Fig. 2. Split spinous process and lamina are opened using a lamina spreader. The ligamentum flavum is split with a microprobe 
Each lamina should be opened gently, little by little, to achieve simultaneous decompression of the entire cervical cord.

Reconstruction of the laminae is performed with a hydroxyapatite (HA) spacer (Figs. 3,4 ), but part of the spinous process or the iliac bone are also possibilities. Using the laminae spreader, the surgeons can measure the size of the spacer to be inserted. Spinal canal enlargement is maintained by placing HA spacers of the appropriate size that are held in place with sutures passed through holes made in the spinous processes. C3-C7 is a typical area for laminoplasty, but a domelike laminotomy of $\mathrm{C} 2$ is added if necessary. To decompress the cord up to $\mathrm{C} 1$, the posterior arch of $\mathrm{C} 1$ is resected.

Before closing the wound, the surgeons should confirm that the dural tube is well decompressed and is not constricted between the split spinous processes. Ultrasonography is useful for confirming that decompression is achieved.

The detached semispinal cervicis muscles are then reattached to the obligus capitis inferior muscles. Preservation of the continuity of the nuchal ligament and reconstruction of the erector spinae muscles to the $\mathrm{C} 2$ are considered important to maintain cervical extensor power and cervical alignment. A suction drainage tube is set over the laminae. The trapezius muscle is tightly sutured with the nuchal ligament, and finally the superficial layers and skin are sutured individually.

\section{Postoperative Management}

The patient is allowed and encouraged to stand up after the suction drain is removed, within 5 days after the operation. A simple cervical collar is used postoperatively for 3 weeks.

\section{Results}

\section{Long-Term Results (More Than 10 Years) of Double-Door Laminoplasty Using Autogenous lliac Bone Grafts}

A total of 60 patients with cervical stenotic myelopathy underwent double-door laminoplasty using an autogenous iliac bone graft between 1980 and 1988 and were

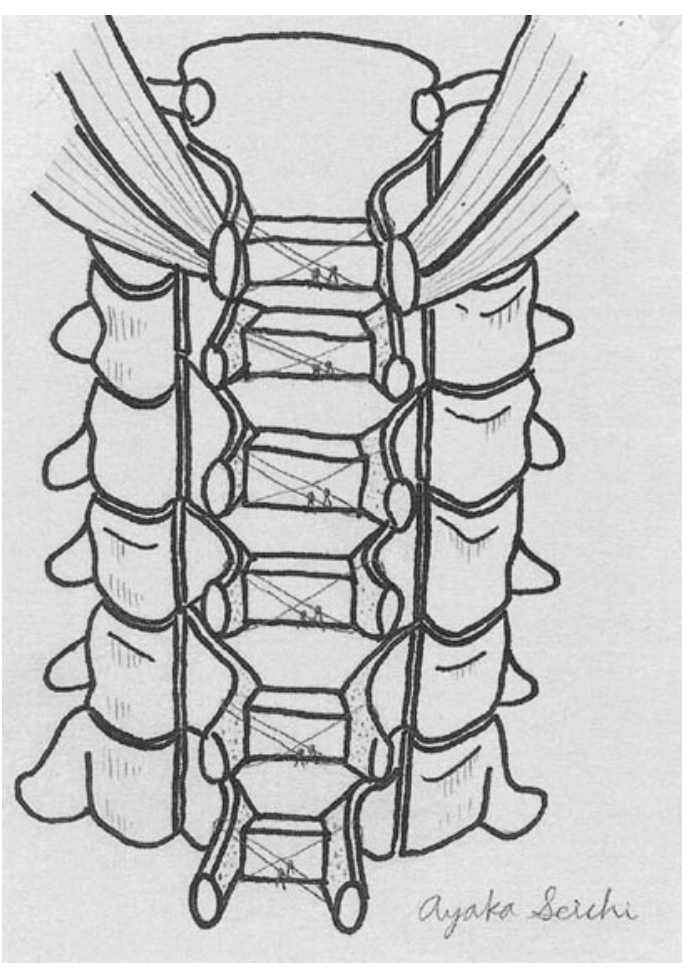

Fig. 3. C1 laminectomy and laminoplasty from C2 to C7 are completed
Fig. 4. Preoperative and postoperative computed tomography
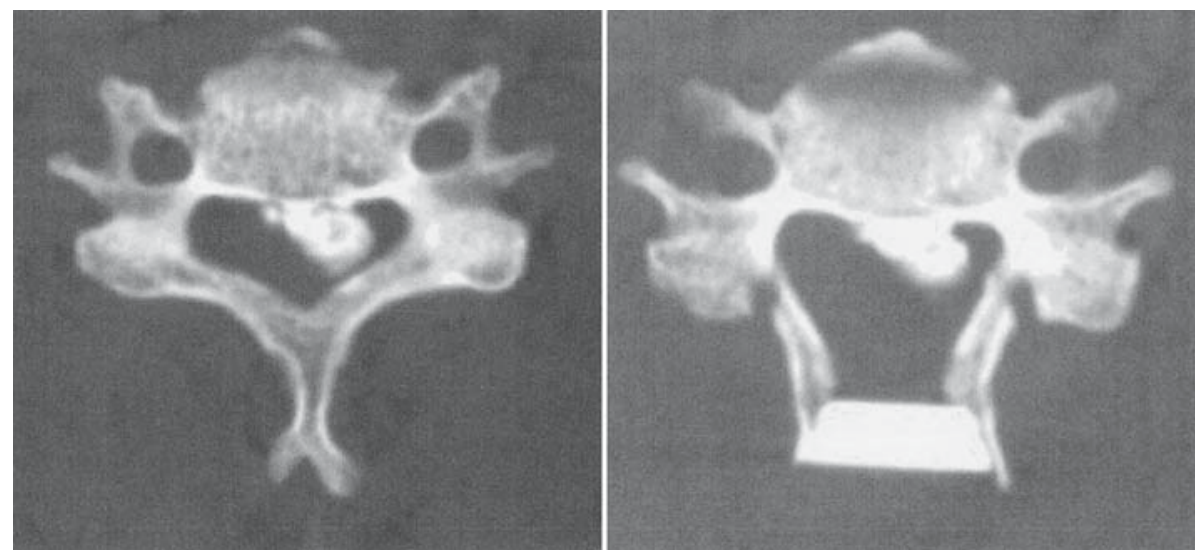
followed for the next 10 years. Of the 60 patients, 35 had cervical myelopathy caused by OPLL in the cervical spine. The average follow-up period was 153 months (range 120-200 months). In this series, one patient used a cervical collar more than 6 weeks.

The severity of their clinical symptoms was assessed using an evaluation score established by the JOA. The results of surgery were estimated by Kurokawa's method based on the scores of motor dysfunction of the upper and lower extremities [13]. In 33 of the 35 patients with OPLL (92\%), the surgical results by Kurokawa's method were fair or better 1 year after surgery (Fig. 5). In 25 of these 35 patients (72\%), the surgical results were maintained until the final followup assessment. Ten patients deteriorated neurologically for an average of 8 years (range 5-12 years). Three deteriorated because of thoracic myelopathy due to ossification of the ligamentum flavum (OLF) of the thoracic spine, which was not directly related to the laminoplasty. These three patients underwent laminectomy of the thoracic spine; and their myelopathy diminished after a second round of surgery in all three. Cervical myelopathy became worse in seven patients, and five of these patients deteriorated after minor trauma (a fall). Their JOA scores were lower after falling, but there was no severe spinal cord injury or loss of walking ability. CT and MRI scans of these five patients showed residual cord compression by OPLL. However, the five had not yet undergone a second round of surgery, such as anterior decompression or fusion. The MRI of the other two did show cervical cord atrophy. The progression of OPLL recognized by lateral radiography was not regarded as a cause of the late deterioration.
The range of motion between $\mathrm{C} 2$ and $\mathrm{C} 7$ was less after surgery, decreasing from a preoperative $36^{\circ}$ to $8^{\circ}$ at final follow-up assessment in all patients and being lost entirely in 11 patients. The unexpected bony fusion of the facet joint on lateral radiography was observed in 30 individuals. The level that had unexpectedly fused most frequently was C2-C3. Neither severe kyphotic deformity nor instability was observed in any case after surgery.

\section{Long-Term Results (More Than 5 Years) of Double-Door Laminoplasty Using Hydroxyapatite Spacers}

A total of 104 patients with cervical stenotic myelopathy underwent double-door laminoplasty using HA spacers between 1989 and 1995. Of these patients, 66 were followed for 5 years after surgery; 31 of them had cervical myelopathy caused by OPLL. In this series, a patient used a cervical collar about 3 weeks to prevent severe loss of cervical spine range of motion. The results of surgery were estimated by Kurokawa's method.

The average follow-up was 78 months (range 60-96 months). At the final follow-up assessment, 8 of the patients had an excellent outcome, 17 were rated good, 5 fair, and 2 poor. The surgical effects of doubledoor laminoplasty using HA spacers were considered to be equivalent to those of double-door laminoplasty using autogenous iliac bone grafts. The range of motion between $\mathrm{C} 2$ and $\mathrm{C} 7$ decreased from a preoperative average of $23^{\circ}$ to $13^{\circ}$ at the final follow-up assessment.

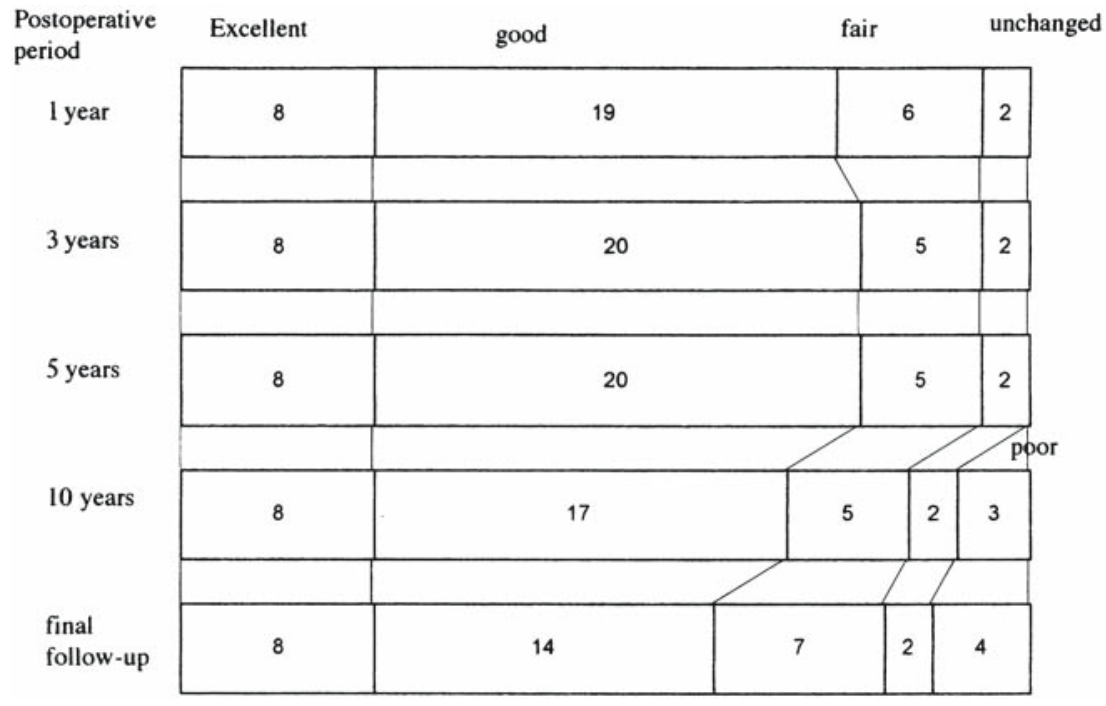

Fig. 5. Changes in the results of surgery (estimated over time by Kurokawa's method) in patients with ossification of the posterior longitudinal ligament who underwent double-door laminoplasty using autogenous iliac bone grafts 


\section{Complications}

\section{Postoperative Paralysis of the Upper Extremities}

Postoperative enlargement of the spinal cord with an intramedullary lesion after decompression surgery for cervical stenotic myelopathy has been reported, but the frequency of the incidence had been unknown. Postoperative motor paresis occurring mainly in the $\mathrm{C} 5$ and C6 segments is also known, but there are various theories about its etiology, including the root involvement hypothesis and the spinal cord impairment hypothesis [16]. To elucidate the prevalence and profile of these complications, the authors conducted a cohort study [17].

A study of all patients who underwent double-door laminoplasty for nontraumatic cervical myelopathy between July 1998 and September 2002 was conducted to investigate the incidence of the postoperative abnormal expansion of T2 high-signal intensity on MRI in the spinal cord and its association with postoperative neurologic deterioration as a short-term complication. Among 124 patients, 2 with a pacemaker for heart disease were excluded because they could not undergo MRI. Three others were excluded because their diagnosis of cervical myelopathy was judged obscure during their postoperative follow-up owing to parkinsonism and other problems. Five patients with cervical spondylotic myelopathy associated with cerebral palsy were also excluded because of poor MRI results. This left 114 patients in the study. The cause of spinal cord compression was cervical spondylosis in 80 patients, OPLL in 21 , rheumatoid arthritis in 8 , cervical spondylosis associated with cerebral palsy in 4 , and calcification of the ligamentum flavum in 1 . All of them underwent preoperative MRI and postoperative MRI 3 weeks after double-door laminoplasty. We watched for the occurrence of postoperative neurologic deterioration including paralysis of the upper extremities. We also observed the presence or absence of postoperative abnormal expansion of T2 high-signal intensity areas on MRI in the spinal cord. Abnormal expansion was defined as preoperative T2 high-signal intensity that spread beyond adjacent intervertebral levels (Fig. 6).

Of the 114 patients, $7(6.1 \%)$, including 3 of the 21 patients with OPLL (14\%) exhibited postoperative abnormal expansion of the T2 high-signal intensity area; three of the seven, including two of the three with OPLL, were asymptomatic. A total of nine patients (7.9\%), including two with OPLL, experienced unilateral upper motor paresis after surgery. In four of the nine cases, paresis of the unilateral deltoid, biceps, and brachialis muscles (proximal paresis) occurred 4-6 days after surgery. None of the four showed postoperative abnormal expansion of the T2 high-signal intensity area. Of the nine patients, three others experienced distal paresis just after surgical intervention. Two of the three exhibited postoperative abnormal expansion of the T2 high-signal intensity area, and one had slight expansion of the area. In the other two cases, diffuse paresis occurred, and their postoperative MRI showed abnormal expansion of the T2 high-signal intensity area. All four patients with proximal paresis had full recovery of their motor loss 3-23 months (average 8 months) after surgery. Two patients with distal paresis had full recovery within 4 and 7 months, and recovery of the other was partial after 16 months. One patient with diffuse paresis showed almost full recovery within 5 months, but recovery of the other individual was only partial at 15 months.

In summary, this investigation showed that spinal cord enlargement with abnormal expansion of the T2 high-signal intensity area, although not common, is an unpreventable complication after laminoplasty. It was strongly related to distal and diffuse types of postoperative paresis of the upper extremity without deterioration of lower motor function, but it was minimally associated with a proximal type of paresis, so-called C5 and C6 palsies, which showed good recovery.

\section{Postoperative Axial Symptoms}

Cervical pain after laminoplasty can correlate with patient satisfaction. The surgical procedure itself and postoperative immobilization have been considered causative factors. We investigated the incidence among patients with cervical spondylotic myelopathy who underwent double-door laminoplasty between September 1998 and May 2005. Of 65 patients with more than 2 years of follow-up, 3 experienced severe pain, 8 moderate pain, and 15 mild pain. We did not make a similar investigation of OPLL patients.

The development of a reasonable method to evaluate postoperative axial symptoms is necessary to learn more about this issue.

\section{Discussion}

Although anterior decompression and fusion, including the floating technique, constitute a radical procedure for OPLL (see the next chapter by Shinomiya et al.), the anterior procedure for multilevel OPLL is highly demanding. A prolonged period of postoperative immobilization of the neck to prevent dislodgement of a long grafted bone is also a burden for patients. Therefore, the posterior approach is often used in Japan. The short-term neurologic recovery was maintained in more than $70 \%$ of the patients with OPLL over a long period. 


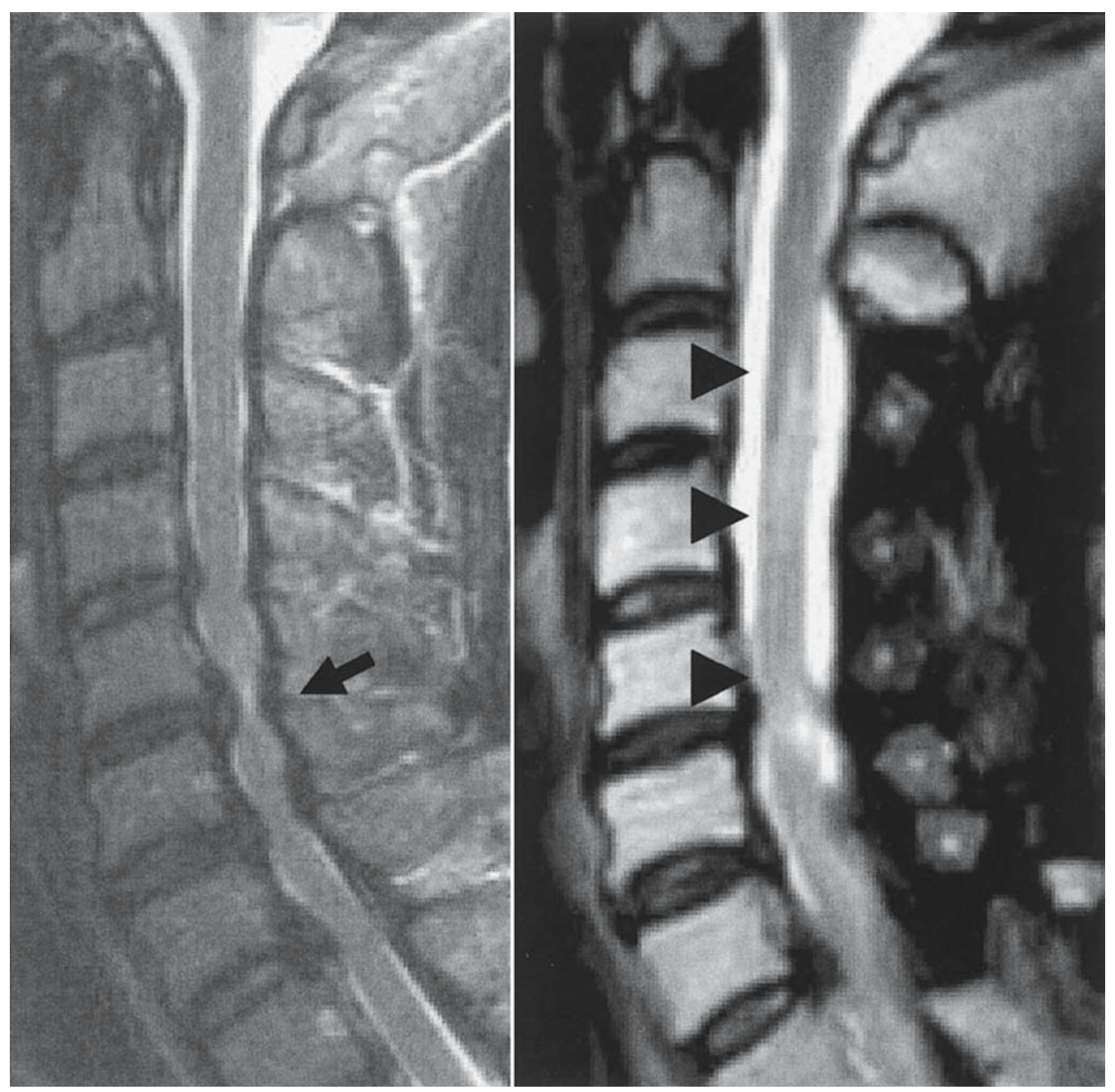

Fig. 6. Preoperative (left) and postoperative (right) T2weighted MR images of a 57-year-old woman with OPLL who developed postoperative abnormal expansion of an intramedullary high-intensity area. Preoperative T2-weighted magnetic resonance imaging (MRI) showed the presence of a highintensity area at C5-C6 (arrow). Postoperative T2-weighted MRI 3 weeks after laminoplasty revealed wide expansion of the high-intensity area between C3 and C5-C6 (arrowheads)
There may be a controversy about a choice of surgery for OPLL [18]: anterior or posterior. We believe that the first choice of surgical treatment for patients with multilevel OPLL is laminoplasty because there are fewer potential risks of a complication. Double-door laminoplasty is a reliable procedure, but a prospective study is necessary to determine the best choice for surgical treatment.

Since 1989, to obtain a good neurological result without severe loss of cervical spine range of motion, we have shortened the postoperative period of neck immobilization and have used HA spacers. This second phase of double-door laminoplasty has improved the resulting range of motion between $\mathrm{C} 2$ and $\mathrm{C} 7$, but the effect was not very good. A tendency toward ossification in patients with OPLL seems to be the main cause.

Surgeons also should know that patients with cervical OPLL often have OPLL or OLF (or both) of the thoracic spine. Careful observation after surgery is therefore necessary for patients with this disorder.

\section{References}

1. Yonenobu K, Okada K, Fuji T, Fujiwara K, Yamashitea K, Ono K (1986) Causes of neurological deterioration follow- 
ing surgical treatment of cervical myelopathy. Spine 11:818-823

2. Mikawa Y, Shikata J, Yamamuro T (1987) Spinal deformity and instability after multilevel cervical laminectomy. Spine 12:6-11

3. Miyazaki K, Kirita Y (1986) Extensive simultaneous multisegmental laminectomy for myelopathy due to the ossification of the posterior longitudinal ligament in the cervical spine. Spine 11:531-542

4. Morimoto T, Okuno S, Nakase H, Kawaguchi S, Sakaki T (1999) Cervical myelopathy due to dynamic compression by the laminectomy membrane: dynamic MR imaging study. J Spinal Dis 12:172-173

5. Kawai S, Sunago K, Doi M, Saika M, Taguchi T (1988) Cervical laminoplasty (Hattori's method): procedure and follow-up results. Spine 13:1245-1250

6. Hirabayashi K, Miyakawa J, Satomi K, Maruyama T, Wakano K (1982) Operative results and postoperative progression of ossification among patients with ossification of cervical posterior longitudinal ligaments. Spine 6:354-364

7. Hirabayashi K, Watanabe K, Wakano K, Suzuki N, Satomi K, Ishii Y (1983) Expansive open-door laminoplasty for cervical spinal stenotic myelopathy. Spine 8: 693-699

8. Kurokawa T, Tsuyama N, Tanaka H, Kobayashi M, Machida H, Izuka T, Hoshino Y, Hatsuyama Y (1982) Enlargement of the spinal canal by the sagittal splitting of the spinous processes (in Japanese). Bessatsu Seikeigeka 2:234-240

9. Heller JG, Edwards CC II, Murakami H, Rodts GE (2001) Laminoplasty versus laminectomy and fusion for multilevel cervical myelopathy: an independent matched cohort analysis. Spine 26:1330-1336

10. Yonenobu K, Fuji T, Ono K, Okada K, Yamamoto T, Harada N (1985) Choice of surgical treatment for multi- segemental cervical spondylotic myelopathy. Spine 10: 710-716

11. Ratriff JK, Cooper PR (2003) Cervical laminoplasty: a critical review. J Neurosurg (Spine 3) 98:230-238

12. Yamazaki A, Homma T, Uchiyama S, Katsumi Y, Okumura H (1999) Morphologic limitations of posterior decompression by midsagittal splitting method for myelopathy caused by ossification of the posterior longitudinal ligament in the cervical spine. Spine 24: 32-34

13. Seichi A, Takeshita K, Ohnishi I, Kawaguchi H, Akune T, Anamizu Y, Kitagawa T, Nakamura K (2001) Long-term results of double-door laminoplasty for cervical stenotic myelopathy. Spine 26:479-487

14. Seichi A, Takeshita K, Kawaguchi H, Higashikawa A, Murakami M, Nakamura K (2005) Intraoperative sonography in laminoplasty for cervical OPLL. In: Investigation committee report on the ossification of the spinal ligament of the Japanese Ministry of Public Health and Welfare and Labour, pp 141-143

15. Takeshita K, Peterson ETK, Bylski-Austrow D, Nakamura $\mathrm{K}$, Crawford AH (2004) The nuchal ligament restrains cervical spine flexion. Spine 29:E388-E393

16. Sakaura H, Hosono N, Mukai Y, Ishii T, Yoshikawa $H$ (2003) C5 palsy after decompression surgery for cervical myelopathy. Spine 28:2447-2451

17. Seichi A, Takeshita K, Kawaguchi H, Nakajima S, Akune T, Nakamura K (2004) Postoperative expansion of intramedullary high-intensity areas on T2-weighted magnetic resonance imaging after cervical laminoplasty. Spine 29:1478-1482

18. Tani T, Ushida T, Ishida K, Iai H, Noguchi T, Yamamoto $\mathrm{H}$ (2002) Relative safety of anterior microsurgical decompression versus laminoplasty for cervical myelopathy with a massive ossified posterior longitudinal ligament. Spine 27:2491-2498 